\title{
PERIODIC SOLUTIONS AND STABILITY OF LINEAR EVOLUTION EQUATIONS WITH NONINSTANTANEOUS IMPULSES
}

\author{
JINRONG WANG AND MICHAL FEČKAN \\ Received 06 March, 2018
}

\begin{abstract}
This paper concerns existence and stability of solutions to periodic linear evolution equations with noninstantaneous impulses via the theory of operator semigroup. A series of fundamental results including compactness, semigroup property, exponential estimate and periodicity are established for a new introduced impulsive evolution operator. Moreover, triple sufficient conditions are given to guarantee this impulsive evolution operator is exponentially stable. In addition, a relationship between existence of periodic solutions and fixed point of impulsive evolution operator is determined, and the alternative results on periodic solutions and their asymptotical stability are obtained by using the well known Fredholm alternative theorem. Finally, an example of periodic impulsive parabolic linear partial differential equation is given for illustration of the theoretically results.
\end{abstract}

2010 Mathematics Subject Classification: 26A33; 93B05

Keywords: linear evolution equations, noninstantaneous impulses, periodic solutions, existence, stability

\section{INTRODUCTION}

Differential equations with instantaneous impulses [5,6,12] have been widely used to characterize the mathematical modeling in the real world life that undergo rapid changes in their state. The duration of these rapid changes is relatively short compared to the overall duration of the whole process. However, there exists another impulsive action in many real world processes, i.e., the evolution process involving abrupt state changes as well as keeping active on a finite time interval.

The first author was supported in part by the National Natural Science Foundation of China (11661016), Training Object of High Level and Innovative Talents of Guizhou Province ((2016)4006), Science and Technology Program of Guizhou Province ([2017]5788-10), and Major Research Project of Innovative Group in Guizhou Education Department ([2018]012).

The second author was supported in part by the Slovak Research and Development Agency under the contract No. APVV-18-0308 and by the Slovak Grant Agency VEGA No. 2/0153/16 and No. $1 / 0078 / 17$. 
Differential equations with non-instantaneous impulses can be used to describe the dynamic of periodic evolution processes in hemodynamic equilibrium of a person. This new class impulsive equation was introduced in [7], which is an extension of standard impulsive differential equations. Owing to the widely application of this new type impulsive equations, there are some generalized, improved, and extended work on this fields, one can see [1-4, 8, 10,13-17] and the reference therein. In fact, Wang and Fečkan [14, p.917] mentioned that the algebraic equation $x=g(t, x)$ in [7] has the only solution depending on $t$, if $g$ satisfied a contraction condition. To overcome this shortcoming, a general class of impulsive evolution equations [14, (1.6)] was established. These systems can be related to differential algebraic equations [11].

In this paper, we consider the following periodic linear non-instantaneous impulsive evolution equations

$$
\left\{\begin{array}{l}
u^{\prime}(t)=A u(t), t \in\left[s_{i}, t_{i+1}\right], i \in \mathbb{N}_{0}:=\{0,1,2, \cdots\}, \\
u\left(t_{i}^{+}\right)=\left(\mathbb{E}+B_{i}\right) u\left(t_{i}^{-}\right)+c_{i}, i \in \mathbb{N}:=\{1,2, \cdots\}, \\
u(t)=\left(\mathbb{E}+B_{i}\right) u\left(t_{i}^{-}\right)+c_{i}, t \in\left(t_{i}, s_{i}\right], i \in \mathbb{N}, \\
u\left(s_{i}^{+}\right)=u\left(s_{i}^{-}\right), i \in \mathbb{N}
\end{array}\right.
$$

where $A: D(A) \subseteq X \rightarrow X$ is the generator of a $C_{0}$-semigroup $\{T(t): t \geq 0\}$ on a Banach space $X$ with a norm $\|\cdot\|, B_{i}: X \rightarrow X, i \in \mathbb{N}$ are bounded linear operators with $B_{i+p}=B_{i}$ and $c_{i} \in X$ with $c_{i+p}=c_{i}$, the $\omega$-periodic time sequences $\left\{t_{i}\right\}_{i \in \mathbb{N}_{0}}$ and $\left\{s_{i}\right\}_{i \in \mathbb{N}_{0}}$ satisfying $t_{i}<s_{i}<t_{i+1}, i \in \mathbb{N}$ with $t_{i+p}=t_{i}+\omega$ and $s_{i+p}=s_{i}+\omega$ where $p \in \mathbb{N}$ denotes the number of impulsive points and connection points of a periodic interval $[0, \omega]$ and set $s_{0}=0$, so $s_{p}=\omega$. Moreover, $\mathbb{E}$ denotes the standard identity operator. We remark that the second equation in (1.1) follows from the third one, but we write it for emphasizing impulses.

Denote $r(t, 0)$ by the number of impulsive points existing in $(0, t)$ and $z_{+}=$ $\max \{0, z\}$ for $z \in \mathbb{R}$. Throughout this paper, we suppose that $T(t) B_{k}=B_{k} T(t)$ for $t>0$.

Consider $U_{T}(\cdot, \cdot):[0, \infty) \times[0, \infty) \rightarrow X$ given by

$$
\begin{aligned}
& U_{T}(t, s) \\
& =T\left(\left(t-s_{r(t, 0)}\right)_{+}-\left(s-s_{r(s, 0)}\right)_{+}+\sum_{k=r(s, 0)}^{r(t, 0)-1}\left(t_{k+1}-s_{k}\right)\right) \prod_{k=r(s, 0)}^{r(t, 0)}\left(\mathbb{E}+B_{k}\right),
\end{aligned}
$$

where we set $\prod_{k=r(s, 0)}^{r(t, 0)-1}\left(\mathbb{E}+B_{k}\right)=\mathbb{E}$ if $r(s, 0)=r(t, 0)$. Obviously,

$$
\begin{aligned}
& U_{T}(t, 0) \\
& =T\left(\left(t-s_{r(t, 0)}\right)_{+}-\left(0-s_{r(0,0)}\right)_{+}+\sum_{k=r(0,0)}^{r(t, 0)-1}\left(t_{k+1}-s_{k}\right)\right) \prod_{k=r(0,0)}^{r(t, 0)}\left(\mathbb{E}+B_{k}\right)
\end{aligned}
$$




$$
=T\left(\left(t-s_{r(t, 0)}\right)++\sum_{k=0}^{r(t, 0)-1}\left(t_{k+1}-s_{k}\right)\right) \prod_{k=0}^{r(t, 0)}\left(\mathbb{E}+B_{k}\right),
$$

and

$$
\begin{aligned}
& U_{T}(\omega, 0) \\
& =T\left(\left(\omega-s_{r(\omega, 0)}\right)_{+}-\left(0-s_{r(0,0)}\right)_{+}+\sum_{k=r(0,0)}^{r(\omega, 0)-1}\left(t_{k+1}-s_{k}\right)\right) \prod_{k=r(0,0)}^{r(\omega, 0)}\left(\mathbb{E}+B_{k}\right) \\
& =T\left(\left(\omega-s_{p}\right)_{+}-\left(0-s_{0}\right)_{+}+\sum_{k=0}^{r(\omega, 0)-1}\left(t_{k+1}-s_{k}\right)\right) \prod_{k=0}^{p}\left(\mathbb{E}+B_{k}\right) \\
& =T\left(\sum_{k=0}^{p-1}\left(t_{k+1}-s_{k}\right)\right) \prod_{k=0}^{p}\left(\mathbb{E}+B_{k}\right) .
\end{aligned}
$$

Introducing

$$
P C_{\omega}([0,+\infty), X)=\{u \in P C([0, \infty), X): u(t)=u(t+\omega), t \geq 0\},
$$

where

$$
\begin{aligned}
P C([0, \infty), X) & =\left\{u:[0, \infty) \rightarrow X: u_{\left.\right|_{i}} \in C\left(J_{i}, X\right), J_{i}=\left(t_{i}, t_{i+1}\right], i \in \mathbb{N}_{0}\right. \\
& \text { and } \left.u\left(t_{i}^{+}\right) \text {and } u\left(t_{i}^{-}\right) \text {exist for each } i \in \mathbb{N}\right\},
\end{aligned}
$$

where $u_{\left.\right|_{J_{i}}}$ denotes the domain of $u$ restricted to the subinterval $J_{i} \subset[0, \infty)$.

The following result is clear.

Theorem 1. The mild solution $u\left(\cdot, u_{0}\right) \in P C([0, \infty), X)$ of $(1.1)$ with the initial condition $u(0)=u_{0}$ has the form

$$
u\left(t, u_{0}\right)=U_{T}(t, 0) u_{0}+\sum_{k=1}^{r(t, 0)} U_{T}\left(t, s_{k}\right) c_{k}, \quad \forall t \geq 0 .
$$

Definition 1. We say the mild solution $u\left(\cdot, u_{0}\right) \in P C([0, \infty), X)$ of $(1.1)$ is $\omega$ periodic if $u\left(t, u_{0}\right)=u\left(t+\omega, u_{0}\right), t \geq 0$.

Definition 2. We say the mild solution $u\left(\cdot, u_{0}\right) \in P C_{\omega}([0,+\infty), X)$ of $(1.1)$ is locally asymptotically stable if there exists a $\delta>0$ such that for any $y_{0} \in X$ with $\left\|u_{0}-y_{0}\right\|<\delta$ such that

$$
\lim _{t \rightarrow \infty}\left\|u\left(t, u_{0}\right)-u\left(t, y_{0}\right)\right\|=0 .
$$

If $\delta$ can be arbitrary then $u\left(\cdot, u_{0}\right) \in P C_{\omega}([0,+\infty), X)$ is globally asymptotically stable. 
Clearly, local asymptotic stability for $\omega$-periodic mild solution of (1.1) coincides with global asymptotic stability.

This paper is organized as follows. Section 2 presents the fundamental properties for the new introduced impulsive evolution operator $U_{T}(\cdot, \cdot)$. The compactness, semigroup property, exponential estimate and periodicity of $U_{T}(\cdot, \cdot)$ as well as exponential stability are obtained for general impulses. Section 3 establishes a relationship between periodic mild solutions of (1.1) and fixed point of $U_{T}(\cdot, \cdot)$ and presents alternative results on periodic solutions of (1.1) and their asymptotical stability. An example is given for illustration of the theoretically results in final section.

\section{BASIC PROPERTIES FOR $U_{T}(\cdot, \cdot)$}

In this section, we study basic properties including compactness, periodicity and exponential stability for (1.2).

We impose the following assumptions:

(H0) Let $A$ be the infinitesimal generator of a $C_{0}$-semigroup $\{T(t): t \geq 0\}$ in $X$. $\left(H 0^{+}\right)\{T(t): t \geq 0\}$ is compact.

(H1) For each $i \in \mathbb{N}, t_{i+p}=t_{i}+\omega$ and $s_{i+p}=s_{i}+\omega$.

(H2) For each $i \in \mathbb{N}, B_{i}: X \rightarrow X$ are bounded linear operators with $B_{i+p}=B_{i}$ and $c_{i+p}=c_{i}$.

By $(H 1)$ and (H2), for each $N \in \mathbb{N}$,

$$
t_{i+N p}=t_{i}+N \omega, s_{i+N p}=s_{i}+N \omega, B_{i+N p}=B_{i}, c_{i+N p}=c_{i} .
$$

Theorem 2. Assumptions $(\mathrm{H} 0),\left(\mathrm{HO}^{+}\right)$hold and suppose $\mathrm{B}_{i}: \mathrm{X} \rightarrow \mathrm{X}$ be bounded linear operators for each $i \in \mathbb{N}$. Then, $U_{T}(t, s)$ is linear and compact for any $\omega \geq t>s \geq 0$.

Proof. The proof directly follows from (1.2), $(\mathrm{H0}),\left(\mathrm{HO}^{+}\right)$and that $B_{i}$ are bounded linear operators.

Theorem 3. The property of semigroup holds for $U_{T}(\cdot, \cdot)$ in $[0, \omega]$, i.e.,

$$
U_{T}(t, s)=U_{T}(t, \tau) U_{T}(\tau, s), 0 \leq s<\tau<t \leq \omega
$$

Proof. Note that the form of (1.2) and the semigroup property of $T(t)$, one can derive that

$$
\begin{aligned}
& U_{T}(t, \tau) U_{T}(\tau, s) \\
& =T\left(\left(t-s_{r(t, 0)}\right)_{+}-\left(\tau-s_{r(\tau, 0)}\right)_{+}+\sum_{k=r(\tau, 0)}^{r(t, 0)-1}\left(t_{k+1}-s_{k}\right)\right) \prod_{k=r(\tau, 0)}^{r(t, 0)}\left(\mathbb{E}+B_{k}\right) \\
& \quad \times \prod_{k=r(s, 0)}^{r(\tau, 0)}\left(\mathbb{E}+B_{k}\right) T\left(\left(\tau-s_{r(\tau, 0)}\right)_{+}-\left(s-s_{r(s, 0)}\right)_{+}+\sum_{k=r(s, 0)}^{r(\tau, 0)-1}\left(t_{k+1}-s_{k}\right)\right)
\end{aligned}
$$


$=U_{T}(t, s)$.

The proof is completed.

Theorem 4. Assumptions (H0), (H1) and (H2) hold. Then, $U_{T}(\cdot, \cdot)$ is $\omega$-periodic, i.e.,

$$
U_{T}(t+N \omega, s+N \omega)=U_{T}(t, s), N \in \mathbb{N} .
$$

Proof. Note that the form of (1.2) and the facts $r(v+N \omega, 0)=r(v, 0)+N p$, one can derive that

$$
\begin{aligned}
& U_{T}(t+N \omega, s+N \omega)=T\left(\left(t+N \omega-s_{r(t+N \omega, 0)}\right)_{+}-\left(s+N \omega-s_{r}(s+N \omega, 0)\right)_{+}\right. \\
& \left.+\sum_{k=r(s+N \omega, 0)}^{r(t+N \omega, 0)-1}\left(t_{k+N p+1}-s_{k+N p}\right)\right)_{k=r(s+N \omega, 0)}^{r(t+N \omega, 0)}\left(\mathbb{E}+B_{k+N p}\right) \\
& =T\left(\left(t+N \omega-\left(s_{r(t, 0)}+N \omega\right)\right)_{+}-\left(s+N \omega-\left(s_{r(s, 0)}+N \omega\right)\right)_{+}\right. \\
& \left.+\sum_{k=r(s, 0)+N p}^{r(t, 0)+N p-1}\left(t_{k+N p+1}-s_{k+N p}\right)\right) \prod_{k=r(s, 0)+N p}^{r(t, 0)+N p}\left(\mathbb{E}+B_{k+N p}\right)=U_{T}(t, s) .
\end{aligned}
$$

The proof is completed.

Theorem 5. Assumptions (H0), (H1) and (H2) hold. Then, we have

$$
U_{T}(t+N \omega, 0)=U_{T}(t, 0)\left[U_{T}(\omega, 0)\right]^{N}, N \in \mathbb{N} .
$$

Proof. We give two proofs as follows:

Method 1. Note that the form of (1.2) and the facts $r(v+N \omega, 0)=r(v, 0)+N p$, one can derive that

$$
\begin{aligned}
& U_{T}(t, 0)\left[U_{T}(\omega, 0)\right]^{N}=T\left(\left(t-s_{r(t, 0)}\right)_{+}+\sum_{k=0}^{r(t, 0)-1}\left(t_{k+1}-s_{k}\right)\right) \prod_{k=0}^{r(t, 0)}\left(\mathbb{E}+B_{k}\right) \\
& \times\left[T\left(\left(\omega-s_{p}\right)_{+}+\sum_{k=0}^{r(\omega, 0)-1}\left(t_{k+1}-s_{k}\right)\right) \prod_{k=0}^{p}\left(\mathbb{E}+B_{k}\right)\right]^{N} \\
& =T\left(\left(t+N \omega-\left(s_{r(t, 0)}+N \omega\right)\right)_{+}+\sum_{k=0}^{r(t, 0)+N p-1}\left(t_{k+N p+1}-s_{k+N p}\right)\right) \\
& \quad \times \prod_{k=0}^{r(t, 0)+N p}\left(\mathbb{E}+B_{k+N p}\right)=U_{T}(t+N \omega, 0) .
\end{aligned}
$$


Method 2. By Theorem 3 and Theorem 4,

$$
\begin{aligned}
U_{T}(t+N \omega, 0) & =U_{T}(t+N \omega, N \omega) U_{T}(N \omega, 0) \\
& =U_{T}(t, 0) U_{T}(N \omega,(N-1) \omega) U_{T}((N-1) \omega, 0) \\
& =U_{T}(t, 0) U_{T}(\omega, 0) U_{T}((N-1) \omega, 0) \\
& \vdots \\
& =U_{T}(t, 0)\left[U_{T}(\omega, 0)\right]^{N}, N \in \mathbb{N} .
\end{aligned}
$$

The proof is completed.

By $(H 0), \exists v \in \mathbb{R}$ and $L \geq 1$ such that

$$
\|T(t)\| \leq L e^{v t}, \forall t \geq 0 .
$$

Theorem 6. For any $0 \leq s<t$,

$$
\begin{aligned}
& \left\|U_{T}(t, s)\right\| \\
& \leq \exp \left\{v\left(\left(t-s_{r(t, 0)}\right)_{+}-\left(s-s_{r(s, 0)}\right)_{+}+\sum_{k=r(s, 0)}^{r(t, 0)-1}\left(t_{k+1}-s_{k}\right)\right)\right\} \\
& \quad \times \exp \left\{\sum_{k=r(s, 0)}^{r(t, 0)} \ln \left\|\mathbb{E}+B_{k}\right\|\right\} .
\end{aligned}
$$

Proof. Taking the norm for (1.2), one can obtain the desired result after some simple calculation.

In the sequel, for general impulses, we suppose

$$
\rho=\sup _{k \geq 1}\left\|\mathbb{E}+B_{k}\right\|<\infty, \quad \mu_{1}=\inf _{k \geq 1}\left(t_{k+1}-s_{k}\right)>0, \quad \mu_{2}=\sup _{k \geq 1}\left(t_{k+1}-s_{k}\right)<\infty
$$

and set

$$
\mu=\left\{\begin{array}{l}
\mu_{1}, v<0 \\
\mu_{2}, v \geq 0
\end{array}\right.
$$

By (2.1), one can derive the following result.

Theorem 7. Assumptions (H0) and (H1) hold. Then it holds

$$
\left\|U_{T}(t, s)\right\| \leq L \rho \exp \{r(t, s)(v \mu+\ln \rho)\} .
$$

Proof. The proof is straightway, so we omit it here.

(H3) Suppose that

$$
\lim _{t-s \rightarrow \infty} \frac{r(t, s)}{t-s}=\sigma<\infty
$$


Theorem 8. Assumptions $(H 0),(H 1)$ and $(H 3)$ hold. Then, $U_{T}(t, s)$ is exponentially stable provided that $\nu \mu+\ln \rho<0$.

Proof. By (H3), for any $1>\epsilon>0$ there exits a $t_{\epsilon}>0$ such that

$$
\left|\frac{r(t, s)}{t-s}-\sigma\right|<\sigma \epsilon, \quad t-s \geq t_{\epsilon},
$$

that is,

$$
(1-\epsilon)(t-s)<\frac{r(t, s)}{\sigma}<(1+\epsilon)(t-s), \quad t-s \geq t_{\epsilon}
$$

Linking (2.3) and (2.4), we derive

$$
\left\|U_{T}(t, s)\right\| \leq L \rho \exp \{\sigma(1-\epsilon)(\nu \mu+\ln \rho)(t-s)\}
$$

for $t-s \geq t_{\epsilon}$. This completes the proof.

(H4) There exists an $\alpha>0$ such that

$$
v+\frac{1}{\mu} \ln \rho \leq-\alpha<0,
$$

where $\mu=\left\{\begin{array}{l}\mu_{1}, \alpha+v<0, \\ \mu_{2}, \alpha+v \geq 0 .\end{array}\right.$

Theorem 9. Assumptions (H0), (H1) and (H4) hold. Then, $U_{T}(t, s)$ satisfies the following estimate:

$$
\left\|U_{T}(t, s)\right\| \leq L \exp \left\{\mu|\alpha+\nu|+\mu_{1} \alpha\right\} \exp \left\{-\alpha \mu_{1} r(s, t)\right\} .
$$

Moreover, $U_{T}(t, s)$ is exponentially stable when (H3) holds.

Proof. Obviously,

$$
\begin{gathered}
\left(t-s_{r(t, 0)}\right)_{+}-\left(s-s_{r(s, 0)}\right)_{+}+\sum_{k=r(s, 0)}^{r(t, 0)-1}\left(t_{k+1}-s_{k}\right) \geq(r(t, s)-1) \mu_{1}, \\
\left(t-s_{r(t, 0)}\right)_{+}-\left(s-s_{r(s, 0)}\right)_{+}+\sum_{k=r(s, 0)}^{r(t, 0)-1}\left(t_{k+1}-s_{k}\right) \leq(r(t, s)+1) \mu_{2} .
\end{gathered}
$$

Linking (2.6) and (2.7), we obtain

$$
\begin{aligned}
\mu_{1}(r(t, s)-1) & \leq\left(t-s_{r(t, 0)}\right)_{+}-\left(s-s_{r(s, 0)}\right)_{+}+\sum_{k=r(s, 0)}^{r(t, 0)-1}\left(t_{k+1}-s_{k}\right) \\
& \leq \mu_{2}(r(t, s)+1),
\end{aligned}
$$


which implies that

$$
\begin{aligned}
& \frac{1}{\mu_{2}}\left(\left(t-s_{r(t, 0)}\right)_{+}-\left(s-s_{r(s, 0)}\right)_{+}+\sum_{k=r(s, 0)}^{r(t, 0)-1}\left(t_{k+1}-s_{k}\right)\right)-1 \\
& \leq r(t, s) \leq \frac{1}{\mu_{1}}\left(\left(t-s_{r(t, 0)}\right)_{+}-\left(s-s_{r(s, 0)}\right)_{+}+\sum_{k=r(s, 0)}^{r(t, 0)-1}\left(t_{k+1}-s_{k}\right)\right)+1 .
\end{aligned}
$$

Then

$$
\begin{aligned}
& -\mu(\alpha+v) r(t, s) \\
& \leq-(\alpha+v)\left(\left(t-s_{r(t, 0)}\right)_{+}-\left(s-s_{r(s, 0)}\right)_{+}+\sum_{k=r(s, 0)}^{r(t, 0)-1}\left(t_{k+1}-s_{k}\right)\right)+\mu|\alpha+v| .
\end{aligned}
$$

Note that $(H 4)$ implies that

$$
-\mu(\alpha+v) r(t, s)=-\sum_{k=r(s, 0)}^{r(t, 0)} \mu(\alpha+v) \geq \sum_{k=r(s, 0)}^{r(t, 0)} \ln \rho \geq \sum_{k=r(s, 0)}^{r(t, 0)} \ln \left\|\mathbb{E}+B_{k}\right\| .
$$

Linking (2.8), (2.9) and (2.10), we obtain

$$
\begin{aligned}
& v\left(\left(t-s_{r(t, 0)}\right)_{+}-\left(s-s_{r(s, 0)}\right)_{+}+\sum_{k=r(s, 0)}^{r(t, 0)-1}\left(t_{k+1}-s_{k}\right)\right)+\sum_{k=r(s, 0)}^{r(t, 0)} \ln \left\|\mathbb{E}+B_{k}\right\| \\
& \leq-\alpha\left(\left(t-s_{r(t, 0)}\right)_{+}-\left(s-s_{r(s, 0)}\right)_{+}+\sum_{k=r(s, 0)}^{r(t, 0)-1}\left(t_{k+1}-s_{k}\right)\right)+\mu|\alpha+v| \\
& \leq-\alpha \mu_{1} r(t, s)+\mu_{1} \alpha+\mu|\alpha+v| .
\end{aligned}
$$

Finally, substituting (2.11) to (2.1), one can derive (2.5).

Linking (2.4) and (2.5), one can verify the exponential stability of $U_{T}(t, s)$ given by

$$
\left\|U_{T}(t, s)\right\| \leq L \exp \left\{\mu|\alpha+v|+\mu_{1} \alpha\right\} \exp \left\{-\alpha \sigma(1-\epsilon) \mu_{1}(t-s)\right\}
$$

for $t-s \geq t_{\epsilon}$. The proof is completed.

Theorem 10. Assume $(H 0)$ and $(H 3)$ hold. Suppose that there is a $p \in[1, \infty)$ such that

$$
\int_{0}^{\infty}\|T(t) x\|^{p} d t<\infty, \forall x \in X,
$$

and

$$
\prod_{k=0}^{\infty}\left\|\mathbb{E}+B_{k}\right\|:=M_{B}<\infty
$$


Then, $U_{T}(t, s)$ is exponentially stable.

Proof. By (2.12) and [9, Theorem 4.1], there are constants $M_{T} \geq 1$ and $v>0$ such that $\|T(t)\| \leq M_{T} e^{-v t}$. Taking the norm for both sides of (1.2) and noticing (2.13), one has

$$
\begin{aligned}
& \left\|U_{T}(t, s)\right\| \\
& \leq M_{T} M_{B} \exp \left\{-v\left[\left(t-s_{r(t, 0)}\right)_{+}-\left(s-s_{r(s, 0)}\right)_{+}+\sum_{k=r(s, 0)}^{r(t, 0)-1}\left(t_{k+1}-s_{k}\right)\right]\right\} \\
& \leq M_{T} M_{B} \exp \left\{\mu_{1} \alpha\right\} \exp \left\{-\alpha \sigma(1-\epsilon) \mu_{1}(t-s)\right\}
\end{aligned}
$$

for $t-s \geq t_{\epsilon}$, which is exponentially stable.

\section{PERIODIC SOLUTIONS AND ASYMPTOTICAL STABILITY}

In this section, we give a sufficient and necessary condition to guarantee that (1.1) has an an $\omega$-periodic mild solution.

Theorem 11. Assumptions (H0), (H1) and (H2) hold. Then (1.3) is an $\omega$ periodic mild solution of (1.1) if and only if $u_{0}$ satisfies

$$
\left(\mathbb{E}-U_{T}(\omega, 0)\right) u_{0}=\sum_{k=1}^{p} U_{T}\left(\omega, s_{k}\right) c_{k} .
$$

Proof. Suppose (1.3) is an $\omega$-periodic mild solution of (1.1), thus, $u\left(t+\omega, u_{0}\right)=$ $u\left(t, u_{0}\right), t \geq 0$ due to Definition 1. Of course, $u_{0}=u\left(\omega, u_{0}\right)$, which implies (3.1), since $r(\omega, 0)=p$. Now, suppose $u_{0} \in X$ solves (3.1). Then (1.3) implies

$$
\begin{aligned}
& u\left(t+\omega, u_{0}\right)=U_{T}(t+\omega, 0) u_{0}+\sum_{k=1}^{r(t+\omega, 0)} U_{T}\left(t+\omega, s_{k}\right) c_{k} \\
& =U_{T}(t+\omega, \omega)\left(U_{T}(\omega, 0) u_{0}+\sum_{k=1}^{p} U_{T}\left(\omega, s_{k}\right) c_{k}\right)+\sum_{k=p+1}^{r(t, 0)+p} U_{T}\left(t+\omega, s_{k}\right) c_{k} \\
& =U_{T}(t, 0) u_{0}+\sum_{k=1}^{r(t, 0)} U_{T}\left(t+\omega, s_{k+p}\right) c_{k+p} \\
& =U_{T}(t, 0) u_{0}+\sum_{k=1}^{r(t, 0)} U_{T}\left(t+\omega, s_{k}+\omega\right) c_{k} \\
& =U_{T}(t, 0) u_{0}+\sum_{k=1}^{r(t, 0)} U_{T}\left(t, s_{k}\right) c_{k}=u\left(t, u_{0}\right),
\end{aligned}
$$


where we use Theorems 3, 4 and properties $r(t+\omega, 0)=r(t, 0)+p, s_{k+p}=s_{k}+\omega$, $c_{k+p}=c_{k}$. The proof is completed.

Next, we give an alternative result.

Theorem 12. Let $(H 0),\left(H 0^{+}\right),(H 1)$ and $(H 2)$ be satisfied. Then one of the following facts is fulfilled:

(i) For any $c_{i} \in X$ with $c_{i+p}=c_{i}$, (1.1) has the unique $\omega$-periodic mild solution. Then $\left[\mathbb{E}-U_{T}(\omega, 0)\right]^{-1}$ exists and it is bounded.

(ii) For any $c_{i} \in X$ with $c_{i+p}=c_{i}$, either (1.1) has no $\omega$-periodic mild solution or it has a finite dimensional set of $\omega$-periodic mild solutions. Then $\left[\mathbb{E}-U_{T}(\omega, 0)\right]^{-1}$ does not exist.

Proof. Under $(\mathrm{HO})$ and $\left(\mathrm{HO}^{+}\right)$, one can see $U_{T}(\omega, 0)$ is linear and compact operator via Theorem 2. The result follows from Theorem 11 by Fredholm Alternative Theorem.

To end this section, we show that $\omega$-periodic mild solution is asymptotically stable.

Theorem 13. Assumptions $(H 0),(H 1),(H 2)$ and $(H 4)$ hold. Then for any $c_{i} \in X$ with $c_{i+p}=c_{i}$, (1.1) has the unique $\omega$-periodic mild solution which is also asymptotically stable.

Proof. Theorem 9 implies that $U_{T}(t, 0)$ is exponentially stable. This gives that the spectral radius of $U_{T}(\omega, 0)$ is less than 1 . Then Neumann lemma leads that $\left[\mathbb{E}-U_{T}(\omega, 0)\right]^{-1}$ exists and it is bounded. Thus Theorem 11 gives the existence and uniqueness result. Next, by Definition 2, it remains to verify that

$$
\lim _{t \rightarrow \infty}\left\|u\left(t, u_{0}\right)-u\left(t, y_{0}\right)\right\|=\lim _{t \rightarrow \infty}\left\|U_{T}(t, 0)\right\|\left\|u_{0}-y_{0}\right\|=0,
$$

which is satisfied again via Theorem 9. The proof is completed.

\section{ApPlication to LINEAR HEAT EQUATIONS}

In this section, we use an example of periodic impulsive parabolic linear partial differential equation to illustrate the above theoretical results.

Set $X=L^{2}(0,1)$ and $s_{0}=0, t_{i}=(2 i-1) \pi, s_{i}=2 i \pi, i \in \mathbb{N}, p=1$ and $\omega=2 \pi$. Obviously, $t_{i+p}=t_{i}+\omega$ and $s_{i+p}=s_{i}+\omega$. 
Consider a parabolic type periodic linear heat equations with non-instantaneous impulses:

$$
\left\{\begin{array}{l}
\frac{\partial}{\partial t} u(t, y)=\kappa^{2} \frac{\partial^{2}}{\partial y^{2}} u(t, y) \\
y \in(0,1), t \in[0, \pi] \cup[2 i \pi,(2 i+1) \pi], i \in \mathbb{N}, \kappa>0 \\
u(t, 0)=u(t, 1)=0, t>0 \\
u\left((2 i-1) \pi^{+}, y\right)=u\left((2 i-1) \pi^{-}, y\right)+b_{i} u\left((2 i-1) \pi^{-}, y\right)+c_{i}(y), i \in \mathbb{N} \\
u(t, y)=u\left((2 i-1) \pi^{-}, y\right)+b_{i} u\left((2 i-1) \pi^{-}, y\right)+c_{i}(y), \\
t \in((2 i-1) \pi, 2 i \pi], i \in \mathbb{N}, \\
u\left(2 i^{+}, y\right)=u\left(2 i^{-}, y\right), i \in \mathbb{N}
\end{array}\right.
$$

for $b_{i} \in \mathbb{R} \backslash\{0\}$ and $c_{i} \in X$. Define $A u=\frac{\partial^{2}}{\partial y^{2}} u$ for $u \in D(A)$ with

$$
D(A)=\left\{u \in X: \frac{\partial u}{\partial y}, \frac{\partial^{2} u}{\partial y^{2}} \in X, u(0)=u(1)=0\right\} .
$$

Then, $A$ is the infinitesimal generator of a $C_{0}$-semigroup $\{T(t), t \geq 0\}$ in $X$.

Let $u_{n}(y)=\sqrt{2} \sin (\pi n y), n=1,2, \cdots$ be the orthonormal set of eigenfunctions of $A$. For any $u \in D(A), A u=\sum_{n=1}^{\infty}\left(-\kappa^{2} n^{2}\right)\left\langle u, u_{n}\right\rangle u_{n}$. Thus,

$$
T(t) u:=\sum_{n=1}^{\infty} \exp \left\{-\kappa^{2} n^{2} t\right\}\left\langle u, u_{n}\right\rangle u_{n},
$$

with $T(\cdot)$ is dissipative and compact with $\|T(t)\| \leq e^{-\kappa^{2} t}$ for all $t \geq 0$. Obviously, $L=1$ and $v=-\kappa^{2}$. Let $B_{i}=b_{i} \mathbb{E}$ and $c_{i}=c_{i}(y)$. Then,

$$
T(\cdot) B_{i}=B_{i} T(\cdot)=\sum_{n=1}^{\infty} b_{i} e^{-\kappa^{2} n^{2} \cdot}\left\langle u, u_{n}\right\rangle u_{n} .
$$

In addition, $(H 0),\left(H 0^{+}\right)$and $(H 1)$ are satisfied. Denoting $u(\cdot)(y)=u(\cdot, y),(4.1)$ can be abstracted into (1.1). Clearly (H3) holds with $\sigma=1$ and $\rho=\sup _{i \in \mathbb{N}}\left|1+b_{i}\right|$, $\mu=\pi$ in (2.2). By (1.2),

$$
\begin{aligned}
& U_{T}(t, s)=\prod_{k=r(s, 0)}^{r(t, 0)}\left(1+b_{k}\right) \\
& \times \sum_{n=1}^{\infty} \exp \left\{-\kappa^{2} n^{2}\left(\left(t-s_{r(t, 0)}\right)_{+}-\left(s-s_{r(s, 0)}\right)_{+}+(r(t, 0)-r(s, 0)) \pi\right)\right\},
\end{aligned}
$$

is exponentially stable provided that

$$
0>v \mu+\ln \rho \Longleftrightarrow \sup _{i \in \mathbb{N}_{0}}\left|1+b_{i}\right|<e^{\pi \kappa^{2}}
$$


via Theorem 8. Next, (2.13) holds either if

$$
\sum_{i=1}^{\infty}\left|b_{i}\right|<\infty, \text { for example, } b_{i}=\frac{1}{i^{2}}
$$

or

$$
\sum_{i=1}^{\infty} \ln \left|1+b_{i}\right|<\infty, \text { for example, } b_{i}= \pm e^{\frac{1}{i^{2}}}-1 .
$$

Then by Theorem 10, (4.2) is exponentially stable.

Now we consider the simplest periodic case, so $b_{1}=b$ and $c_{1}=c$ for any $i \in \mathbb{N}$. Then obviously,

$$
U_{T}(\omega, 0) u=(1+b) \sum_{n=1}^{\infty} e^{-\kappa^{2} n^{2} \pi}\left\langle u, u_{n}\right\rangle u_{n}
$$

and

$$
u\left(\omega, u_{0}\right)=(1+b) \sum_{n=1}^{\infty} e^{-\kappa^{2} n^{2} \pi}\left\langle u_{0}, u_{n}\right\rangle u_{n}+c(y) .
$$

So (3.1) becomes

$$
\left(1-(1+b) e^{-\kappa^{2} n^{2} \pi}\right) \bar{u}_{n}=\bar{c}_{n}, \quad n \in \mathbb{N},
$$

where $u_{0}=\sum_{n=1}^{\infty} \bar{u}_{n} u_{n}$ and $c=\sum_{n=1}^{\infty} \bar{c}_{n} u_{n}$. From (4.4), we deduce: If

$$
e^{\kappa^{2} n^{2} \pi} \neq 1+b, \quad \forall n \in \mathbb{N},
$$

then the alternative (i) of Theorem 12 holds for (4.1): There is a unique $2 \pi$-periodic mild solution of (4.1) given by

$$
u\left(t, u_{0}\right)=\left\{\begin{array}{l}
\sum_{n=1}^{\infty} \frac{e^{-\kappa^{2} n^{2} t} \bar{c}_{n}}{1-(1+b) e^{-\kappa^{2} n^{2} \pi}} u_{n} \quad t \in[0, \pi] \\
\sum_{n=1}^{\infty}\left(\frac{(1+b) e^{-\kappa^{2} n^{2} \pi}}{1-(1+b) e^{-\kappa^{2} n^{2} \pi}}+1\right) \bar{c}_{n} u_{n} \quad t \in(\pi, 2 \pi] .
\end{array}\right.
$$

If

$$
e^{\kappa^{2} \widetilde{n}^{2} \pi}=1+b
$$

for some $\tilde{n} \in \mathbb{N}$, then the alternative (ii) of Theorem 12 holds for (4.1): If $\bar{c}_{\tilde{n}} \neq 0$ then there is no a $2 \pi$-periodic mild solution of (4.1), but if $\bar{c}_{\tilde{n}}=0$ then there is a 1-dimensional space $\left[u_{\tilde{n}}\right]$ of $2 \pi$-periodic mild solutions of (4.1).

Next, (4.3) becomes

$$
|1+b|<e^{\pi \kappa^{2}},
$$

which implies (4.5). For example, we choose $b=0.05$ satisfying $|1+0.05|<$ $e^{0.02 \pi} \approx 1.0648$.

Hence then there is a unique $2 \pi$-periodic mild solution of (4.1), which is in addition asymptotically stable (see Figures 1-3). 


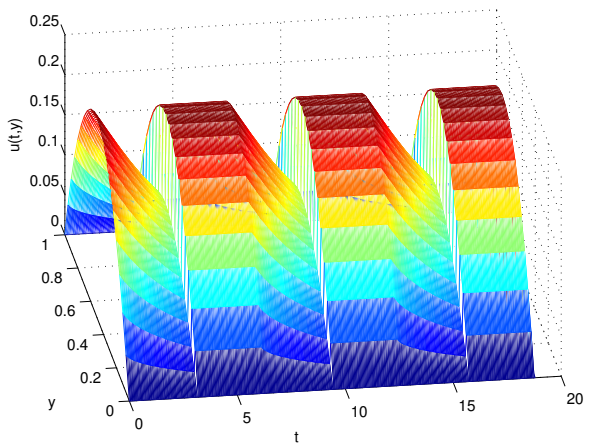

FIGURE 1. The periodic solution of (4.1) with $\kappa^{2}=0.02, u_{0}(y)=y(1-$ $y), y \in[0,1], b_{i}=0.05$.

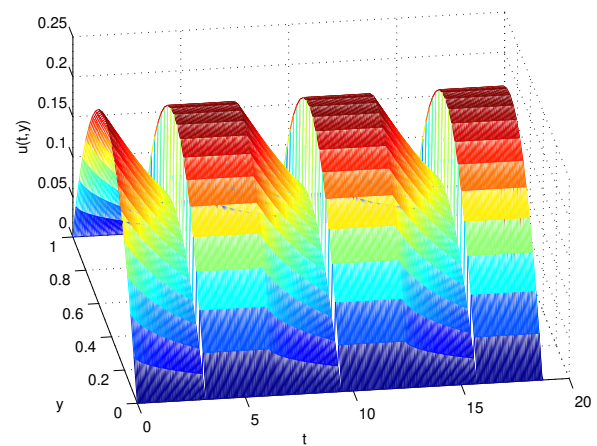

FIGURE 2. The periodic solution of (4.1) with $\kappa^{2}=0.02, u_{0}(y)=$ $1.01 y(1-y), y \in[0,1], b_{i}=0.05$.

Note that for $b_{i}=b,(4.4)$ gives

$$
c_{i}(y)=c(y)=\sum_{n=1}^{\infty}\left(1-(1+b) e^{-\kappa^{2} n^{2} \pi}\right) \bar{u}_{n} u_{n}(y)
$$

knowing $u_{0}(y)$. For instance, if $u_{0}(y)=y(1-y)$, then using the Fourier series of the function $y(1-y)$ with respect to the orthogonal basis $\sin \pi n y$, we have

$$
u_{0}(y)=\frac{8}{\pi^{3}} \sum_{n=1}^{\infty} \frac{\sin (2 n+1) \pi y}{(2 n+1)^{3}}=\sum_{n=1}^{\infty} \frac{4 \sqrt{2}}{(2 n+1)^{3} \pi^{3}} u_{2 n+1}(y),
$$

so

$$
\bar{u}_{2 n+1}=\frac{4 \sqrt{2}}{(2 n+1)^{3} \pi^{3}}, \quad \bar{u}_{2 n}=0,
$$




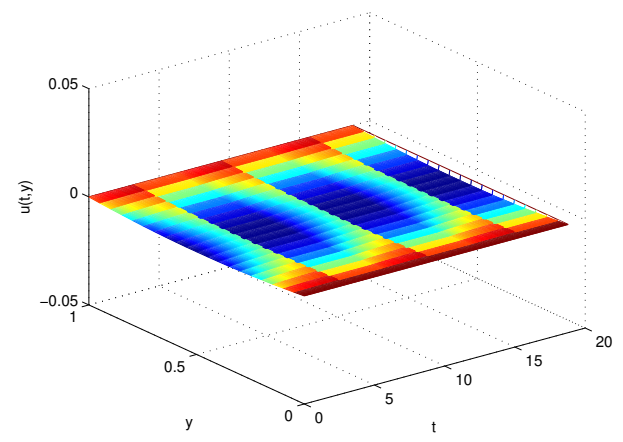

FIGURE 3. The difference between the Figures 1 and 2.

and

$$
\begin{aligned}
c(y) & =\sum_{n=1}^{\infty} \frac{4 \sqrt{2}\left(1-(1+b) e^{-\kappa^{2} n^{2} \pi}\right)}{(2 n+1)^{3} \pi^{3}} u_{2 n+1}(y) \\
& =\frac{8}{\pi^{3}} \sum_{n=1}^{\infty} \frac{\left(1-(1+b) e^{-\kappa^{2} n^{2} \pi}\right) \sin (2 n+1) \pi y}{(2 n+1)^{3}} .
\end{aligned}
$$

For numerical computation, it is useful to use also another formula

$$
c=u_{0}-(1+b) u\left(\pi, u_{0}\right) .
$$

\section{ACKNOWLEDGMENT}

The authors thank the referees for carefully reading the manuscript and for their valuable comments.

\section{REFERENCES}

[1] S. Abbas and M. Benchohra, "Uniqueness and Ulam stabilities results for partial fractional differential equations with not instantaneous impulses." Appl. Math. Comput., vol. 257, pp. 190-198, 2015, doi: 10.1016/j.amc.2014.06.073.

[2] R. Agarwal, D. O'Regan, and S. Hristova, "Non-instantaneous impulses in Caputo fractional differential equations." Frac. Calc. Appl. Anal., vol. 20, no. 3, pp. 595-622, 2017, doi: 10.1515/fca2017-0032.

[3] R. Agarwal, D. O'Regan, and S. Hristova, "Noninstantaneous impulses in Caputo fractional differential equations and practical stability via Lyapunov functions." J. Franklin Inst., vol. 354, no. 7, pp. 3097-3119, 2017, doi: 10.1016/j.jfranklin.2017.02.002.

[4] R. Agarwal, D. O’Regan, and S. Hristova, "Stability with initial time difference of Caputo fractional differential equations by Lyapunov functions.” Z. Anal. Anwend., vol. 36, no. 1, pp. 49-77, 2017, doi: 10.4171/ZAA/1579.

[5] D. D. Bainov and P. S. Simeonov, Impulsive Differential Equations: Periodic Solutions and Applications. Harlow: Chapman and Hall, 1993. 
[6] M. Benchohra, J. Henderson, and S. K. Ntouyas, Impulsive Differential Equations and Inclusions. Cairo: Hindawi Publishing Corporation, 2006.

[7] E. Hernández and D. O'Regan, "On a new class of abstract impulsive differential equation." Proc. Amer. Math. Soc., vol. 141, no. 5, pp. 1641-1649, 2013, doi: 10.1090/S0002-9939-2012-11613-2.

[8] E. Hernández, M. Pierri, and D. O'Regan, "On abstract differential equations with non instantaneous impulses." Topol. Meth. Nonlinear Anal., vol. 46, no. 2, pp. 1067-1085, 2015, doi: 10.12775/TMNA.2015.080.

[9] Z. Pazy, Semigroup of Linear Operatiors and Applications to Partial Differential Equations. New York: Springer, 1983. doi: 10.1007/978-1-4612-5561-1.

[10] M. Pierri, D. O'Regan, and V. Rolnik, "Existence of solutions for semi-linear abstract differential equations with not instantaneous impulses." Appl. Math. Comput., vol. 219, no. 12, pp. 67436749, 2013, doi: 10.1016/j.amc.2012.12.084.

[11] R. Riaza, Differential-Algebraic Systems, Analytical Aspects and Circuit Applications. Singapore: World Scien. Publ. Co. Pte. Ltd., 2008. doi: https://doi.org/10.1142/6746.

[12] A. M. Samoilenko and N. A. Perestyuk, Impulsive Differential Equations. Singapore: World Scien. Publ. Co. Pte. Ltd., 1995. doi: https://doi.org/10.1142/2892.

[13] J. Wang, "Stability of noninstantaneous impulsive evolution equations." Appl. Math. Lett., vol. 73, pp. 157-162, 2017, doi: 10.1016/j.aml.2017.04.010.

[14] J. Wang and M. Fečkan, "A general class of impulsive evolution equations." Topol. Meth. Nonlinear Anal., vol. 46, no. 2, pp. 915-934, 2015, doi: 10.12775/TMNA.2015.072.

[15] J. Wang, M. Fečkan, and Y. Tian, "Stability analysis for a general class of non-instantaneous impulsive differential equations." Mediter. J. Math., vol. 14, no. 2, pp. 1-21, 2017, doi: 10.1007/s00009-017-0867-0.

[16] D. Yang, J. Wang, and D. O'Regan, “A class of nonlinear non-instantaneous impulsive differential equations involving parameters and fractional order." Appl. Math. Comput., vol. 321, pp. 654-671, 2018, doi: 10.1016/j.amc.2017.11.025.

[17] D. Yang, J. Wang, and D. O'Regan, "On the orbital Hausdorff dependence of differential equations with non-instantaneous impulses.” C. R. Acad. Sci. Paris, Ser. I, vol. 356, no. 2, pp. 150-171, 2018 , doi: 10.1016/j.crma.2018.01.001.

Authors' addresses

JinRong Wang

JinRong Wang, Department of Mathematics, Guizhou University, Guiyang, Guizhou 550025, P.R. China, and Qufu Normal University, School of Mathematical Sciences, Qufu, Shandong 273165, P.R. China

E-mail address: jrwang@gzu.edu.cn

\section{Michal Fečkan}

Michal Fečkan, Department of Mathematical Analysis and Numerical Mathematics, Faculty of Mathematics, Physics and Informatics, Comenius University, Mlynská dolina, 84248 Bratislava, Slovakia, and Mathematical Institute, Slovak Academy of Sciences, Štefánikova 49, 81473 Bratislava, Slovakia

E-mail address: Michal.Feckan@ fmph.uniba.sk 